\title{
Resolving stellar populations outside the Local Group: MAD observations of UKS 2323-326^
}

\author{
1 Osservatorio Astronomico di Padova, INAF, vicolo dell'Osservatorio 5, 35122 Padova, Italy \\ e-mail: marco.gullieuszik@oapd.inaf.it \\ 2 Osservatorio Astronomico di Bologna, INAF, via Ranzani 1, 40127 Bologna, Italy \\ ${ }^{3}$ European Southern Observatory, Karl-Schwarzschild-Strasse 2, 85748 Garching, Germany
}

M. Gullieuszik ${ }^{1}$, L. Greggio ${ }^{1}$, E. V. Held ${ }^{1}$, A. Moretti ${ }^{1}$, C. Arcidiacono ${ }^{1}$, P. Bagnara ${ }^{1}$, A. Baruffolo ${ }^{1}$, E. Diolaiti ${ }^{2}$, R. Falomo ${ }^{1}$, J. Farinato ${ }^{1}$, M. Lombini ${ }^{2}$, R. Ragazzoni ${ }^{1}$, R. Brast ${ }^{3}$, R. Donaldson ${ }^{3}$, J. Kolb ${ }^{3}$, E. Marchetti ${ }^{3}$, and S. Tordo ${ }^{3}$

Received 22 February 2008 / Accepted 18 March 2008

\section{ABSTRACT}

\begin{abstract}
Aims. We present a study aimed at deriving constraints on star formation at intermediate ages from the evolved stellar populations in the dwarf irregular galaxy UKS 2323-326. These observations were also intended to demonstrate the scientific capabilities of the multi-conjugated adaptive optics demonstrator (MAD) implemented at the ESO Very Large Telescope as a test-bench of adaptive optics (AO) techniques.

Methods. We perform accurate, deep photometry of the field using $J$ and $K_{\mathrm{s}}$ band AO images of the central region of the galaxy. Results. The near-infrared (IR) colour-magnitude diagrams clearly show the sequences of asymptotic giant branch (AGB) stars, red supergiants, and red giant branch (RGB) stars down to $\sim 1$ mag below the RGB tip. Optical-near-IR diagrams, obtained by combining our data with Hubble Space Telescope observations, provide the best separation of stars in the various evolutionary stages. The counts of AGB stars brighter than the RGB tip allow us to estimate the star formation at intermediate ages. Assuming a Salpeter initial mass function, we find that the star formation episode at intermediate ages produced $\sim 6 \times 10^{5} M_{\odot}$ of stars in the observed region.
\end{abstract}

Key words. galaxies: individual: UKS2323-326 - galaxies: stellar content - stars: AGB and post-AGB - stars: carbon instrumentation: adaptive optics - galaxies: individual: ugca438

\section{Introduction}

The study of the resolved stellar populations in external galaxies has developed greatly in the last decade to become arguably the most accurate tool to investigate star formation history in stellar systems. However, with standard instrumentation at groundbased telescopes this study is limited to the nearest galaxies, due to the severe crowding of stars. High-precision photometry for the most distant galaxies in the Local Group (LG) and beyond can be obtained only with the Hubble Space Telescope (HST).

New opportunities in this field are foreseen with the realisation of imagers equipped with adaptive optics (AO), on the largest aperture telescopes. The use of $\mathrm{AO}$ systems is mandatory for the future larger $(>10 \mathrm{~m})$ telescopes, but it can also significantly improve the performances of telescopes already in operation. A relevant example is given by the multi-conjugated adaptive optics demonstrator (MAD) recently developed by ESO (see next section) that allows us to test AO capabilities for stellar photometry on the sky.

In this context, as part of a Guaranteed Time Observations program, we obtained MAD near-infrared (IR) images of the dwarf irregular galaxy UKS 2323-326 (UGCA 438). We chose this galaxy from a list of targets selected according to various criteria: favourable position on the sky with respect to the

\footnotetext{
* Based on observations collected at the European Southern Observatory, Chile, as part of MAD Guaranteed Time Observations, on ESO archival observations (Programme 71.D-0560), and on
} NASA/ESA Hubble Space Telescope observations (Proposal ID 8192). availability of stars to perform the AO correction; low Galactic latitude $(b=-70.9)$, to minimise the contamination by foreground Galactic stars; location slightly beyond the boundary of the LG, so as to maximise the physical area sampled within the $1^{\prime}$ field-of-view (FoV) while still detecting stars at the tip of the red giant branch (TRGB) with an adequate $\mathrm{S} / \mathrm{N}$; existence of images of the same field in HST and/or ESO archives; and the presence of a relatively strong intermediate age component.

Currently, AO imagers operate only at near-IR wavelengths, which are best suited to studying evolved stellar populations, in particular, cool stars on the asymptotic giant branch (AGB). This evolutionary stage of low and intermediate mass stars is difficult to model because of its sensitivity to uncertain input physics, like mass loss and convection. However, AGB stars provide a major contribution to the integrated light of galaxies with intermediateage stellar populations (Renzini \& Buzzoni 1986), therefore, it is very important to derive information on the productivity of these stars. This can be done by analysing the stellar content of galaxies with a strong intermediate age component, which is the motivation for our near-IR study of LG galaxies (Held et al. 2007; Gullieuszik et al. 2007a,b).

Ground-based optical photometry of UKS 2323-326 was first presented by Lee \& Byun (1999). The colour-magnitude diagram (CMD) exhibits a well-defined RGB, and a number of AGB stars. From the TRGB magnitude and from the colour of these stars, Lee \& Byun (1999) derive the distance modulus and average metallicity of the galaxy as $(m-M)_{0}=26.59 \pm$ 0.12 and $[\mathrm{Fe} / \mathrm{H}]=-1.98$. More recently, from photometry 
obtained with the WFPC2 on board of the HST, Karachentsev et al. (2002) measured $I^{\mathrm{TRGB}}=22.72 \pm 0.12$, from which they obtain $(m-M)_{0}=26.74 \pm 0.15$, corresponding to $2.23 \pm$ $0.15 \mathrm{Mpc}$. Thus, this galaxy is likely a member of the Sculptor Group. Since the HST photometry is more accurate and since the distance determination by Karachentsev et al. (2002) is based on a more modern calibration, in this paper, we will adopt the Karachentsev et al. (2002) value. This implies that the absolute magnitude of the galaxy is $M_{V}=-13.24$. Although UKS 2323-326 contains a young stellar component, there is no evidence of significant H II emission (Miller 1996; Kaisin et al. 2007), which suggests a very low rate of ongoing star formation. No mid-infrared emission from hot dust nor polycyclic aromatic hydrocarbon is detected at $8 \mu \mathrm{m}$ (Jackson et al. 2006) but it is embedded in a neutral hydrogen cloud that asymmetrically covers the whole galaxy (e.g., Buyle et al. 2006). The H I mass is $\sim 6 \times 10^{6} M_{\odot}$, while for CO emission only an upper limit on the molecular gas mass of $1.4 \times 10^{5} M_{\odot}$ is available (Buyle et al. 2006, ans refs. therein).

\section{The data}

\subsection{MAD observations}

MAD is a project (Marchetti et al. 2007) mainly developed by ESO to test the multi-conjugated adaptive optics (MCAO) capabilities on the sky in the framework of the design of the European Extremely Large Telescope (ELT). MAD was mounted on the UT-3 of the Very Large Telescope (VLT) to realise the first MCAO observation on the sky (Bouy et al. 2008). The instrument accommodates two wavefront sensors (WFS): a star-oriented multi-Shack-Hartmann and a layer-oriented (LO, Ragazzoni et al. 2000; Vernet-Viard et al. 2005) multi-pyramid (Ragazzoni 1996). Both WFS use reference stars on a 2' technical FoV. MAD is complemented with the CAMCAO scientific IR camera, with a $2 k \times 2 k$ Hawaii II IR detector that can be moved across the $2^{\prime}$ corrected circular FoV. The pixel scale is 0 . $^{\prime} 028$ pixel $^{-1}$, yielding a $57^{\prime \prime} \times 57^{\prime \prime}$ square FoV on the detector.

We took observations of UKS 2323-326 on Sep. 27, 2007 with the LO wavefront sensor option, with the aim of testing single pyramid AO observations in the bright-end regime. The reference star has $V \approx 11.5$ and is located at $\sim 24^{\prime \prime}$ from the centre of the FoV. This is the very first pyramid WFS AO-assisted science observation (see Ragazzoni \& Farinato 1999, for a discussion of the advantages of this technique) on an $8 \mathrm{~m}$-class telescope. Results from other observations with full multi-pyramid MCAO capabilities will be presented elsewhere.

Our data set consists of $21 \mathrm{~J}$ frames and $15 K_{\mathrm{S}}$ frames centred on UKS 2323-326, at $\alpha(\mathrm{J} 2000)=23^{\mathrm{h}} 26^{\mathrm{m}} 27^{\mathrm{s}}, \delta(\mathrm{J} 2000)=$ $-32^{\circ} 23^{\prime} 16^{\prime \prime}$. The total integration time is 37 and $30 \mathrm{~min}$ in $J$ and $K_{\mathrm{s}}$ band, respectively.

\subsection{Reduction and photometric calibration}

We calibrated our near-IR photometry by comparing stars in common with the 2MASS point-source catalogue (Skrutskie et al. 2006) and by using $J$ and $K_{\mathrm{s}}$ archive images obtained with the Son of ISAAC (SOFI) camera mounted at the ESO New Technology Telescope (NTT) to define secondary photometric standards.

We reduced both SOFI and MAD raw images following the standard procedure for IR data, as described by Gullieuszik et al. (2007a). We paid careful attention to image alignment, allowing a correction for possible field rotation. We limited the area used in the final analysis to a $45^{\prime \prime} \times 45^{\prime \prime}$ region because of stray light affecting one edge of the MAD images. From the integration of the $R$-band surface brightness profile (Lee \& Byun 1999) of the galaxy, we estimate that the luminosity fraction observed is $\sim 36 \%$.

The point spread function (PSF) of stellar objects on the combined MAD images is fairly uniform across the whole frame, with deviations of $\lesssim 10 \%$ of the full width at half maximum (FWHM). The mean FWHM measured on the $J$ an $K_{\mathrm{s}}$ images is, respectively, 0.15 and $0{ }^{\prime} 11$. This is a good result, considering that the seeing was 0.52 and 0.42 in $J$ and $K_{\mathrm{s}}$, respectively (estimated from the ESO DIMM Monitor measurements in the $V$ band) and that the diffraction limit for the VLT is 0. '04 for the $J$ band and 0. . 07 for the $K_{\mathrm{s}}$ band. The Strehl ratio measured on the $J$ and $K_{\mathrm{s}}$ frames is $7.6 \%$ and $21.4 \%$, respectively. The ellipticity of stellar images is small (9\% and $10 \%$ in $J$ and $K_{\mathrm{s}}$ ), with an rms variation $\lesssim 5 \%$ over the whole FoV.

We performed stellar photometry on MAD and SOFI images using DAOPHOT/ALLSTAR programs (Stetson 1987), adopting a Penny model for the PSF, with a quadratic dependence on the position on the frame. The astrometric and photometric calibration of the SOFI data was obtained using the USNO-A2.0 (Monet et al. 1998) and the 2MASS (Skrutskie et al. 2006) databases. We then used the resulting catalogue as a reference for the astrometric and photometric calibration of MAD frames. Considering the uncertainty of our two-steps calibration, the final error on the zero-point of MAD photometry resulted $\simeq 0.15$ mag.

Finally, the near-IR data were complemented with optical WFPC2/HST data from Holtzman et al. (2006). This allows us to test the spatial resolution of MAD images, as well as take advantage of a wide colour baseline to study the stellar population.

Figure 1 compares the 3 images from SOFI, MAD, and WFPC2 for the same region in UKS 2323-326. The improvement in resolution between MAD and SOFI image is clearly apparent. This implies a significantly better photometry of faint objects and, in particular, the possibility of obtaining accurate photometry for faint stars that are embedded in the halo of brighter stars.

In order to evaluate the completeness and photometric errors of our catalogue, we performed an extensive set of 160 artificial star experiments using 1000 stars for each run. Input magnitudes were randomly generated to reproduce an uniform distribution over the colour and magnitude range of real stars in our image $\left(0<J-K_{\mathrm{s}}<2\right.$ and $\left.15<K_{\mathrm{s}}<22.5\right)$. We found that our photometry is complete at the $50 \%$ level down to $K_{\mathrm{S}} \simeq 20.7$. At this magnitude level, the photometric error is $\sim 0.1$ mag in the $K_{\mathrm{s}}$ band, and a factor of 2 lower in the $J$ band. These results are illustrated in Fig. 2.

\section{The evolved stellar populations of UKS 2323-326}

The CMD of UKS 2323-326 obtained from MAD images is shown in Fig. 2 and compared to that obtained from SOFI data in the same FoV. The MAD CMD is about 1 mag deeper; more importantly, the photometric accuracy is higher, leading to a much better definition of the sequences on the CMD. This is mostly due to the higher spatial resolution of MAD, which allows us to resolve stars that are blended on SOFI images.

The red tail of bright stars in the MAD CMD, extending up to $J-K_{\mathrm{S}} \simeq 1.8$ and $K_{\mathrm{S}} \simeq 18.5$, is consistent with the locus of carbon-rich AGB stars (cf. other recent near-IR studies, e.g., Gullieuszik et al. 2007a,b; Menzies et al. 2008). It is tempting 

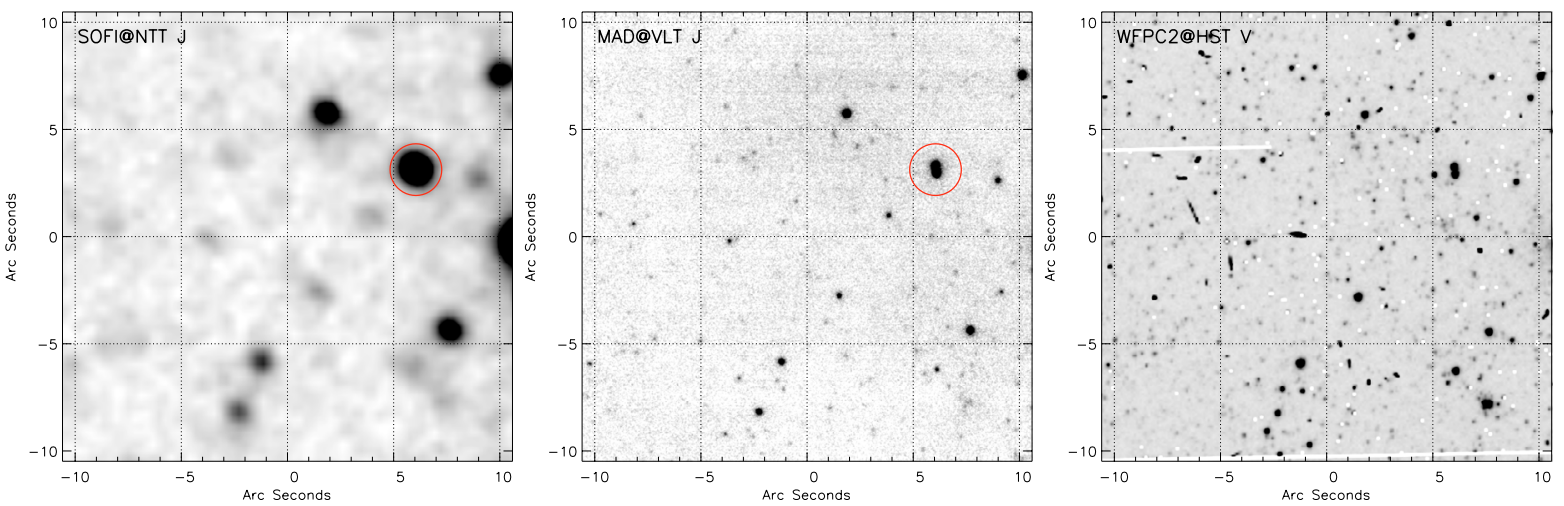

Fig. 1. SOFI, MAD and WFPC2 images of the same region in UKS 2323-326. Only a section of the full area analysed in our study is shown to better illustrate the details. The two circles highlight the effect of the higher spatial resolution of MAD versus SOFI.

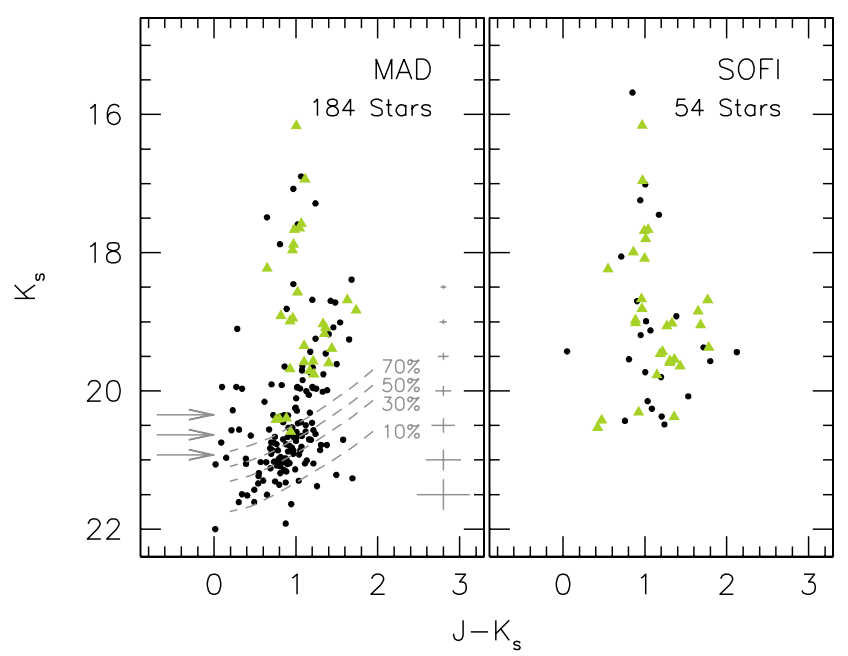

Fig. 2. CMD obtained from MAD data (left) and from SOFI data (right) for the same FoV. In the left panel, the completeness levels (dashed lines) and photometric errors (crosses) from artificial star experiments are shown. The expected location of the TRGB is indicated with arrows for $[\mathrm{Fe} / \mathrm{H}]=-1.0,-1.5$, and -2.0 (top to bottom). Stars used as secondary photometric standards are shown as triangles.

to locate the TRGB at $K_{\mathrm{s}} \simeq 20.5$, where a discontinuity is apparent in the stellar magnitude distribution. However, a formal measurement of the TRGB from the luminosity function cannot be derived because of the incompleteness of our photometry at these magnitudes. Since the distance modulus is known from optical observations, we can estimate the expected level of the TRGB: according to Valenti et al. (2004) calibration, the absolute $K_{\mathrm{S}}$ magnitude of the TRGB depends on metallicity, being $-5.82,-6.11$, and -6.40 for $[\mathrm{Fe} / \mathrm{H}]=-2,-1.5$, and -1 , respectively. Assuming a reddening $E_{B-V}=0.015$ (Schlegel et al. 1998 ), we get $K_{\mathrm{s}}^{\mathrm{TRGB}}=20.93,20.64$, and 20.35 , as the metallicity increases. These three values are indicated with arrows in Fig. 2: for the metallicity determined by Lee \& Byun (1999) $([\mathrm{Fe} / \mathrm{H}] \simeq-2)$, the TRGB should be close to the limit of our photometry. The Lee \& Byun (1999) determination is based on a relatively shallow CMD. Comparing the deeper $(V, I)$ HST CMD by Holtzman et al. (2006) to the fiducial lines of Galactic globular clusters by Da Costa \& Armandroff (1990), we obtain a mean metallicity $[\mathrm{Fe} / \mathrm{H}] \simeq-1.7$ for the RGB stars in this galaxy. This has to be regarded as a lower limit, since the bulk of UKS 2323-326 stellar population is younger than Galactic globular clusters. As an example, Saviane et al. (2000) estimated that

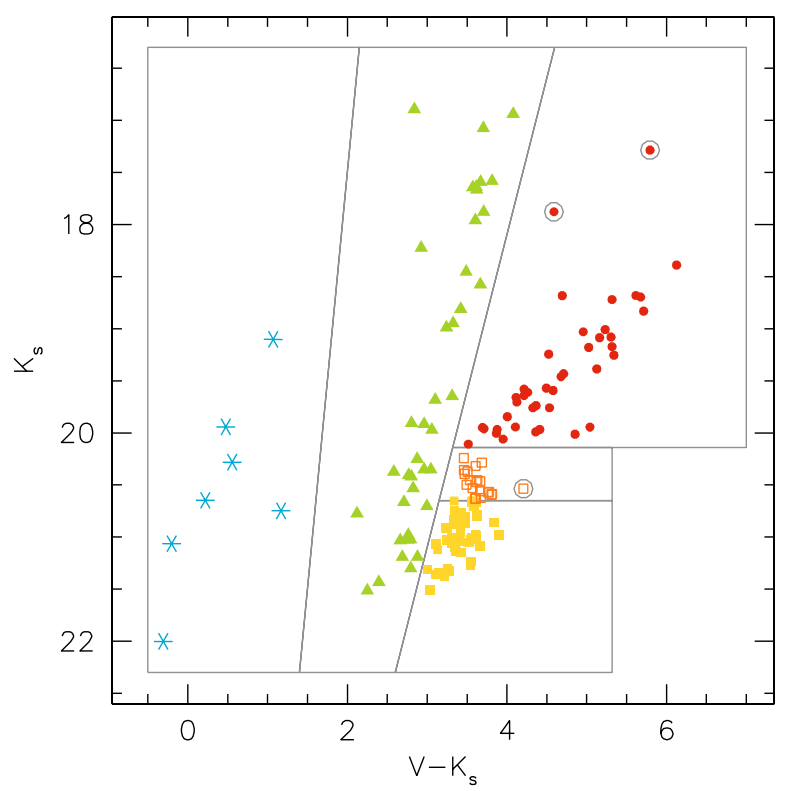

Fig. 3. Combined optical-near-IR MAD CMD of UKS 2323-326. We used this CMD to select blue supergiants (starred symbols), red supergiants (triangles), RGB (squares), and AGB stars (filled circles). Open squares are the stars with an uncertain classification, but likely E-AGB stars (see text for details). The three stars marked by open circles have peculiar colours and are possibly photometric blends.

for a $\sim 5$ Gyr stellar population, the age correction to be applied to the metallicity obtained with our method is +0.4 dex. The location of the TRGB would then come close to the discontinuity of the star's distribution mentioned above. In the following, we adopt $[\mathrm{Fe} / \mathrm{H}]=-1.5$, which yields a TRGB magnitude of $K_{\mathrm{s}}=20.65$; we verified that this metallicity is compatible with the mean $V-K_{\mathrm{s}}$ colour of the RGB in UKS 2323-326.

Figure 3 shows the $V-K_{\mathrm{s}}$ vs. $K_{\mathrm{s}} \mathrm{CMD}$ obtained by combining the HST and MAD photometry. This colour combination is particularly well suited to distinguish the different evolutionary sequences. The contamination by foreground stars in our fields is negligible. In fact, using simulations of the Milky Way population performed with the TRILEGAL code (Girardi et al. 2005), we expect only three foreground stars in the magnitude and colour range of our CMD. Guided by the optical CMD, on this figure we draw the lines bordering the areas occupied by blue supergiants, red supergiants, AGB, and RGB stars. The blue and red supergiants are core Helium burning stars with masses 


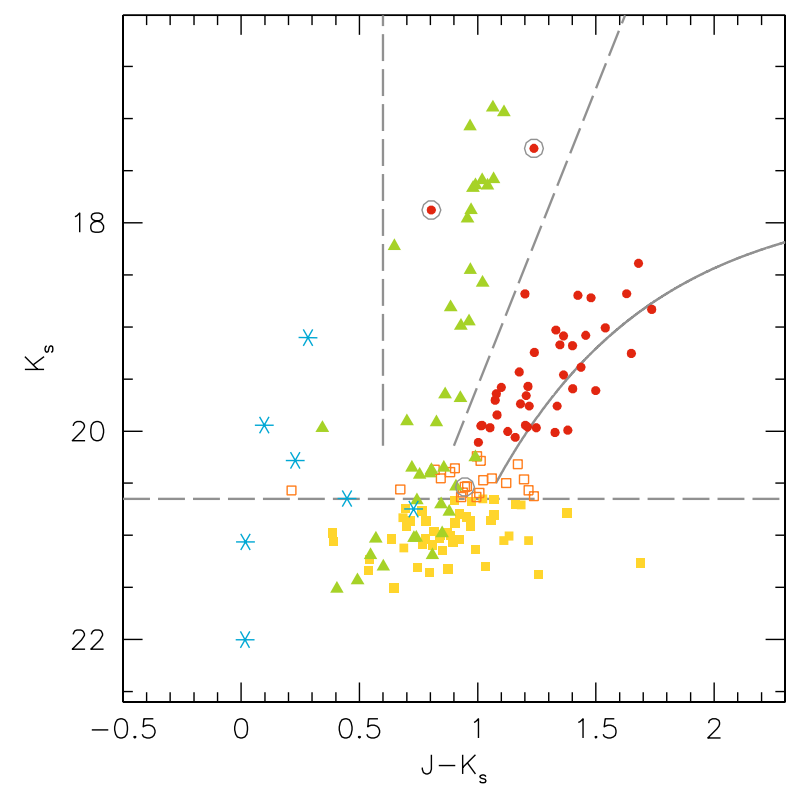

Fig. 4. The near-IR CMD of UKS 2323-326. The different symbols refer to the selection presented in Fig. 3. The solid line is the main locus of C-star in nearby dwarf galaxies defined by Totten et al. (2000) shifted to the adopted distance of UKS 2323-326.

down to $\sim 5 M_{\odot}$, resulting from the star formation activity occurred over the last $\sim 100 \mathrm{Myr}$. The main-sequence progenitors of this young population are sampled in the optical CMD by Holtzman et al. (2006), in which the blue plume contains stars with ages from $\simeq 80$ to $\simeq 10 \mathrm{Myr}$ old. A quantitative interpretation of this component needs detailed simulations that take into account photometric errors and completeness of the HST data. Here we concentrate on the intermediate age component for which the near-IR CMD offers a better diagnostic than the optical diagram. The bright portion of the red sequence, extending from $K_{\mathrm{s}} \simeq 20.1$ up to $K_{\mathrm{s}}=18.5$ and with very red colours (up to $V-K_{\mathrm{s}}=6.5$ ) hosts bright AGB, mostly carbon (C) stars, while below the TRGB (at $K_{\mathrm{s}}=20.65$ ) we sample the oldest stars. The stars in the intermediate region (open squares, $20.65>K_{\mathrm{s}}>20.1$ ) are probably AGB stars, but some of them could actually be high-metallicity RGB stars. The uncertainty stems from the dependence of the TRGB $K_{\mathrm{S}}$-magnitude on the metallicity discussed above, and on these stars being located (on the optical CMD) just below the TRGB in the $I$ band.

Our observations can be used to derive a rough estimate of the star formation that occurred in UKS 2323-326 at intermediate ages by considering the number of AGB stars brighter than the TRGB, which is proportional to the gas mass converted into stars between $\sim 0.1$ and a few Gyr (e.g., Greggio 2002). The specific production of bright AGB stars (i.e., $\delta n_{\mathrm{AGB}, \mathrm{b}}$, the number of stars per unit mass of the parent stellar population that fall in this region of the CMD) depends on age and metallicity. We have determined $\delta n_{\mathrm{AGB}, \mathrm{b}}$ as a function of age, for a sample of globular clusters in the Large Magellanic Cloud for which nearIR CMDs, ages, and total luminosities are known from the literature. From these, we found that it reaches a maximum $(\sim 2 \times$ $10^{-4} M_{\odot}^{-1}$ ) at ages $\sim 1 \mathrm{Gyr}$, to drop significantly at older ages, down to $\sim 3 \times 10^{-5} M_{\odot}^{-1}$ at $\sim 3$ Gyr (close to the limit of our calibration). The stellar distribution in our CMD is suggestive of an extended episode of star formation; by averaging our empirical calibration, we obtain a specific production of 0.15 or 0.08 stars per $10^{3} M_{\odot}$, if this episode started 1.5 or $3 \mathrm{Gyr}$ ago, respectively.
Since we count 59 objects on the red sequence at magnitudes brighter than the TRGB, we bracket the stellar mass produced at intermediate ages in the range between 4 and $7.5 \times 10^{5} M_{\odot}$. We note that these estimates are based on a straight Salpeter initial mass function (IMF). If a Chabrier (2005) IMF were assumed, the masses would be $\sim 0.65$ times smaller.

Using the classification obtained from the $\left(V, K_{\mathrm{s}}\right) \mathrm{CMD}$, we construct the $\left(J, K_{\mathrm{s}}\right)$ diagram to see how the various sequences can be identified when only IR data are available (see Fig. 4). Although the sequences here are less well traced, the various evolutionary stages are relatively well separated. In particular, this CMD shows that all stars in the red tail are located in the position expected for $\mathrm{C}$-stars, since they are compatible with the main locus of $\mathrm{C}$-stars in nearby dwarf galaxies defined by Totten et al. (2000). These results confirm that near-IR CMDs are a very powerful tool to clearly detect C-stars, as indicated by our previous studies of LG galaxies (Gullieuszik et al. 2007a,b).

To summarise our work, we obtained a complete and accurate census of the bright evolved stellar population (red supergiants and AGB stars) in UKS 2323-326, a dwarf irregular galaxy at a distance of $2.23 \mathrm{Mpc}$. We have shown that with nearIR AO images at $8 \mathrm{~m}$ class telescopes it is possible to investigate the SFH in galaxies well beyond the LG. Considering the technical limitation of the demonstrator, we believe that these results forecast very promising opportunities for this kind of studies with advanced AO at ELT.

\section{References}

Bouy, H., Kolb, J., Marchetti, E., et al. 2008, A\&A, 477, 681

Buyle, P., Michielsen, D., de Rijcke, S., Ott, J., \& Dejonghe, H. 2006, MNRAS, 373,793

Chabrier, G. 2005, in The Initial Mass Function 50 Years Later, ed. E. Corbelli, F. Palla, \& H. Zinnecker, ASSL, 327, 41

Da Costa, G., \& Armandroff, T. 1990, AJ, 100, 162

Girardi, L., Groenewegen, M. A. T., Hatziminaoglou, E., \& da Costa, L. 2005, A\&A, 436, 895

Greggio, L. 2002, in Observed HR Diagrams and Stellar Evolution, ed. T. Lejeune, \& J. Fernandes, ASP Conf. Ser., 274, 444

Gullieuszik, M., Held, E. V., Rizzi, L., et al. 2007a, A\&A, 467, 1025

Gullieuszik, M., Rejkuba, M., Cioni, M. R., Habing, H. J., \& Held, E. V. 2007b, A\&A, 475, 467

Held, E. V., Gullieuszik, M., Rizzi, L., et al. 2007, in From Stars to Galaxies: Building the Pieces to Build Up the Universe, ed. A. Vallenari, R. Tantalo, L. Portinari, \& A. Moretti, ASPC Ser., 374, 261

Holtzman, J. A., Afonso, C., \& Dolphin, A. 2006, ApJS, 166, 534

Jackson, D. C., Cannon, J. M., Skillman, E. D., et al. 2006, ApJ, 646, 192

Kaisin, S. S., Kasparova, A. V., Knyazev, A. Y., \& Karachentsev, I. D. 2007, Astron. Lett., 33, 283

Karachentsev, I. D., Sharina, M. E., Makarov, D. I., et al. 2002, A\&A, 389, 812 Lee, M. G., \& Byun, Y.-I. 1999, AJ, 118, 817

Marchetti, E., Brast, R., Delabre, B., et al. 2007, The Messenger, 129, 8

Menzies, J., Feast, M., Whitelock, P., et al. 2008, MNRAS, 385, 1045

Miller, B. W. 1996, AJ, 112, 991

Monet, et al. 1998, VizieR Online Data Catalog, I/252

Ragazzoni, R. 1996, J. Mod. Opt., 43, 289

Ragazzoni, R., \& Farinato, J. 1999, A\&A, 350, L23

Ragazzoni, R., Farinato, J., \& Marchetti, E. 2000, in Adaptive Optical Systems Technology, ed. P. L. Wizinowich, Proc. SPIE, 4007, 1076

Renzini, A., \& Buzzoni, A. 1986, in Spectral Evolution of Galaxies, ed. C. Chiosi, \& A. Renzini, ASSL, 122, 195

Saviane, I., Held, E. V., \& Bertelli, G. 2000, A\&A, 355, 56

Schlegel, D. J., Finkbeiner, D. P., \& Davis, M. 1998, ApJ, 500, 525

Skrutskie, M. F., Cutri, R. M., Stiening, R., et al. 2006, AJ, 131, 1163

Stetson, P. B. 1987, PASP, 99, 191

Totten, E. J., Irwin, M. J., \& Whitelock, P. A. 2000, MNRAS, 314, 630

Valenti, E., Ferraro, F. R., \& Origlia, L. 2004, MNRAS, 354, 815

Vernet-Viard, E., Arcidiacono, C., Bagnara, P., et al. 2005, Optical Engineering, 44,6601 